EGU21-7658, updated on 05 May 2021

https://doi.org/10.5194/egusphere-egu21-7658

EGU General Assembly 2021

(c) Author(s) 2021. This work is distributed under

the Creative Commons Attribution 4.0 License.

\title{
Atomic oxygen in the mesosphere and lower thermosphere measured by terahertz heterodyne spectroscopy
}

\author{
Heinz-Wilhelm Hübers ${ }^{1,2}$, Heiko Richter ${ }^{1}$, Christof Buchbender ${ }^{3}$, Rolf Güsten ${ }^{4}$, Ronan Higgins ${ }^{3}$, \\ Bernd Klein $^{4,5}$, Jürgen Stutzki ${ }^{3}$, and Helmut Wiesemeyer ${ }^{4}$ \\ ${ }^{1}$ German Aerospace Center (DLR), Institute of Optical Sensor Systems, Berlin, Germany \\ ${ }^{2}$ Humboldt-Universität zu Berlin, Department of Physics, Berlin, Germany \\ ${ }^{3}$ I. Physikalisches Institut der Universität zu Köln, Cologne, Germany \\ ${ }^{4}$ Max-Planck-Institut für Radioastronomie, Bonn, Germany \\ ${ }^{5}$ University of Applied Sciences Bonn-Rhein-Sieg, Sankt Augustin, Germany
}

Atomic oxygen is a main component of the mesosphere and lower thermosphere (MLT). The photochemistry and the energy balance of the MLT are governed by atomic oxygen. In addition, it is a tracer for dynamical motions in the MLT. It is difficult to measure with remote sensing techniques. Concentrations can be inferred indirectly from the oxygen air glow or from observations of $\mathrm{OH}$, which is involved in photochemical processes related to atomic oxygen. Such measurements have been performed with several satellite instruments such as SCIAMACHY, SABER, WINDII and OSIRIS. However, the methods are indirect and rely on photochemical models and assumptions such as quenching rates, radiative lifetimes, and reaction coefficients. The results are not always in agreement, particularly when obtained with different instruments.

We have explored an alternative approach, namely the observation of the ${ }^{3} \mathrm{P}_{1}->{ }^{3} \mathrm{P}_{2}$ fine-structure transition of atomic oxygen at $4.7 \mathrm{THz}(63 \mu \mathrm{m})$ using the German Receiver for Astronomy at Terahertz Frequencies (GREAT) on board of SOFIA, the Stratospheric Observatory for Infrared Astronomy. GREAT is a heterodyne spectrometer providing high sensitivity and high spectral resolution as low as $76 \mathrm{kHz}$. This method enables the direct measurement without involving photochemical models to derive the atomic oxygen concentration. The night-time measurements have been performed during a SOFIA flight along the west coast of the US. These are the first measurements which resolve the line shape of the 4.7-THz transition. From the spectra the concentration profiles and radiances of atomic oxygen were derived with a radiative transfer model. The observed radiances range from 1.5 to $2.2 \mathrm{nW} \mathrm{cm}^{-2} \mathrm{sr}^{-1}$ and the the altitude profiles agree within the measurement uncertainty with SABER data and the NRLMSISE-00 model [1].

In conclusion, $\mathrm{THz}$ heterodyne spectroscopy is a powerful method to measure atomic oxygen in the MLT. With the current progress in $\mathrm{THz}$ technology balloon-borne and space-borne $4.7-\mathrm{THz}$ heterodyne spectrometers become feasible [2,3]. Combining such a $\mathrm{THz}$ spectrometer with optical instruments similar to SABER or SCIAMACHY will be even more advantageous for the determination of atomic oxygen in the MLT. 
[1] H. Richter et al., Direct measurements of atomic oxygen in the mesosphere and lower thermosphere using terahertz heterodyne spectroscopy, accepted for publication in Communications Earth \& Environment (2021).

[2] M. Wienold et al, A balloon-borne 4.75 THz-heterodyne receiver to probe atomic oxygen in the atmosphere, to appear in: Proceedings of the $45^{\text {th }}$ International Conference on Infrared, Millimeter, and Terahertz Waves (IRMMW-THz) (Buffalo, NY, 2020).

[3] S. P. Rea et al., The low-cost upper-atmosphere sounder (LOCUS), Proceedings of the 26th International Symposium on Space Terahertz Technology (Cambridge, MA, 2015). 